\title{
Controlling the operating conditions in an Operating Room
}

\author{
C. Rosa, A. Costa, E. Fernandes, F. Cardoso, P. S. Guimarães, J. Alves, I. Silva \& M. A. Marques \\ Physics Department, Instituto Superior de Engenharia do Porto (ISEP), Porto, Portugal
}

\begin{abstract}
In this paper, we describe the development of a module capable of controlling environmental conditions, personnel movement and management of other important parameters for an operating room (OR) or surgery block. The base of assembly uses a real-life small scale model trying to simulate the systematic functionality of an $O R$ as well as its crucial components. These include the regulation of environmental parameters, logging of entries and exits by the staff, management of pharmaceutical drugs and diseases, and the statistical analysis of several acquired data. The project integrates different types of technologies, such as the microcontroller Arduino, a bidirectional communication interface between this device and a computer, a web based user interface for dynamic interaction, as well as database concepts for data management.
\end{abstract}

Index Terms - Operating Room, Surgery Block, Control, Arduino, Environmental Conditions, Web Interface.

\section{INTRODUCTION}

A $\mathrm{n}$ operating room is a facility where a great diversity of services and medical specialties meet together to provide medical assistance and care to patients. Operating rooms can be used for either urgent or programed surgical interventions, or even for delicate medical exams. Normally categorized by having large sterilized, well illuminated rooms in order to provide optimal brightness to avoid shadowing effects and parallax errors that may induce the surgeon in a mistake. Operating Rooms are therefore, facilities with very high levels of technical demand, either at the structural and equipment level, or at the procedure and execution level [1-2].

Sterilization is an essential service of an Operating Room. Therefore, it's important to control the in/out flow related to clean/used material or sterilized/contaminated equipment [2].

Access to the room is controlled by using credentials for authentication, consequently only authorized pers onnel or members related to a certain medical specialty are free to enter. Additionally, it's important to control the number of people already inside, and deny additional accesses in order to avoid unnecessary crowds in the same space all at once, this is done by efficiently controlling the staff ingoing and outgoing.

Another important as pect is the medication distribution management. This service has to be prepared to respond quickly to any request while it maintains a regular and steady supply in response to any emerging need in critical situations, like for example, during a surgical intervention [3]. Rigorous control of the environmental conditions is also one of the biggest concerns in operating rooms, due to their sensitive setting. Parameters like, temperature atmospheric pressure, light intensity and air flow, are determinant for a surgical intervention [4]. The recommended temperature interval during an operation rages from $18^{\circ} \mathrm{C}$ to $23^{\circ} \mathrm{C}$ [5], which is primarily defined for surgeon comfort, and to avoid fatigue considering the long time periods for certain type of surgeries. The room temperature should be monitored, and it's controlled regularly. In this situation, our model uses a heating device and Peltier plates. The relative humidity interval lies between $20 \%$ and $60 \%$ (ANSI/ASHRAE/ASHE Standard 170-2008). The equipment used to control this variable is the dehumidifier. Concerning lighting, the recommended average level is about 300 lux, when in simple observation, and 1000 lux for surgical procedures or treatments [5].

\section{SYSTEM HARDWARE AND FEATURES}

$\mathrm{T}$ he model developed for this work, took into account the control of all parameters described previously. The microcontroller Arduino is used as the basis for most components, especially to read all sensors, accept input from the keyboard, display information on the various displays and to control the servo-motors. A picture of the model is shown in Figure 1.

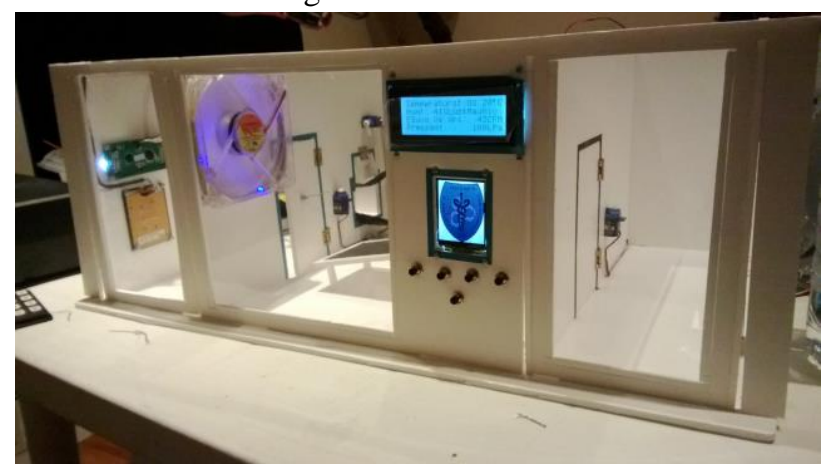

Figure 1: Picture of the physical structure of the model

\section{A. Sensors for environmental conditions}

Temperature was measured using an analog output sensor, the LM35. Humidity was measured using a DHT11. The atmospheric pressure inside the room is monitored using the barometric sensor, the BMP085. Air flow was simulated using a $120 \mathrm{~m} 12 \mathrm{~V}$ brushless fan. Light level fluctuation was measured using Light Dependent Resistors (LDR). Each one of these parameters has a trigger point, which means that a certain event can be fired in order to stabilize a corresponding parameter. For example, if temperature falls down the operating interval that was defined previously, a heater will be turned on until temperature falls within the expected interval. On the other hand, if temperature is above 
operational interval, a cooler based on the Peltier effect, is turned on to bring the temperature down to its normal value. All of these parameters are shown in a color LCD. Data is also transmitted to the database using the communication interface developed. The measured values are analyzed to detect any abrupt changes in any of the sensors. This is done to test if any of the sensors was removed, if any of them sustained physical damage. For example, if the temperature sensor has to be removed for whichever reason, the system will detect this error and will display it in the LCD which will turn red. It will also $\log$ any of these errors in the database.

\section{B. Operating features}

As stated before, only authorized personnel may have access to the operating room and its interfaces. This access is done sequentially using a personal ID code, similar to the login in the web interface, and followed by the presentation of an ID card. The codes are introduced via a keyboard and a card reader based on a RFID module. If authentication is successful, the doors will open, giving access to a sterilization room. After a certain time, considered necessary for the sterilizing process to finish, the operating room doors open.

The medication system is operated by the health professional in charge of this task. The medication ID is introduced in the requisition keypad as well as the desired quantity. This process will then activate a pulley contraption that will carry the desired medication from the outside of the room, where pharmaceutical storage room may be located, to the inside of the operating room. Every medication request will be then logged in the database. It's necessary to provide the user with sufficient information to optimize the operating room conditions, like for example how many persons are inside the room at a certain instant and their categories, i.e., number of doctors, nurses or anaesthesiologists, etc.

\section{WEB INTERFACE ANDCOMMUNICATION SOFT WARE}

The web interface was developed with the intent to give detailed information about the multiple parameters described. The interface allows the management of data related to user registration, medication and drugs in stock, intervention/surgery scheduling, supervision of the several environmental conditions, and finally statistics such as the medication cost of an intervention. These functionalities are accessible by the interface window shown in Figure 2.

The programming languages used to develop the web interface include PHP, JavaScript (with AJAX), as well MySQL. The database is structured in a way to allow the following operations:

- User management: login, passwords (with encryption), RFID codes;

- Logging of environmental conditions, sensor errors, faults, power cuts, as well as their duration.

- Surgery scheduling and its final state, indicating if it was a successful or if it resulted in decease of the patient.

- Relationship between different diseases to which surgeries can be as sociated with;

- Logging of personnel circulation with timestamp information.

- Management of medication and drug storage;

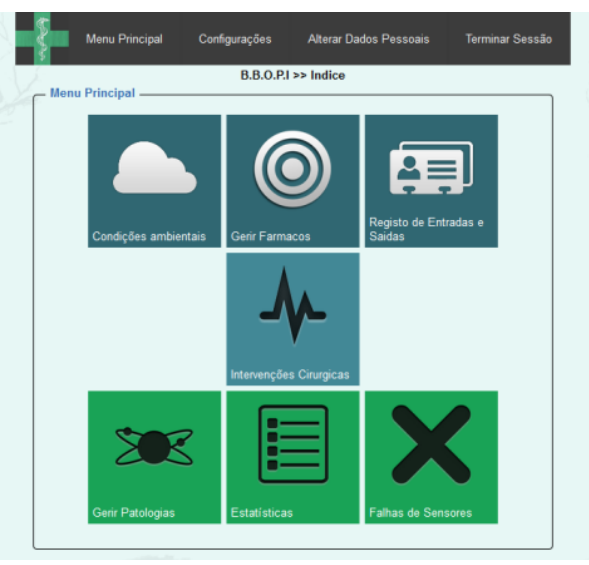

Figure 2: Web interface used to access the several functionalities

Communication with the microcontroller Arduino required a bidirectional interface with the database that allowed it to read and write information. The communication interface was programmed in C\# along with MySQL's ODBC driver.

\section{CONCLUSIONS}

$\mathrm{T}$ he proposed features for controlling the operating room conditions were successfully achieved. Since this was a semi-large scale project, where different members of our group worked in different modules simultaneously, special care was taken while providing guidelines, writing source code, all to avoid misguided instructions. All modules were tested independently before assembly which resulted in a less time spent in this task. As stated earlier, this work considers some of the most critical parameters, but a lot more could be implemented in future iterations. This project was developed as an academic module, but taking in consideration the features and functionalities implemented, there is certainly a possibility to continue this work somehow. In conclusion this project demonstrates the importance of controlling certain parameters within an operating room model, which can be applied in real life.

\section{REFERENCES}

[1] Poore SO, Sillah NM, Mahajan AY, Gutowski KA, "Patient safety in the operating room: II. Intraoperative and postoperative.”, 2012 Nov.

[2] UONIE/ACSS do Sistema de Saúde, "Recomendações Técnicas para Bloco Operatório", RT 05/2011, http://www.acss.minsaude.pt

[3] American Society of Anesthesiologists, "Statement on Security of Medications in the Operating Room", October 17, 2012.

[4] Brock L, "The importance of environmentalconditions, especially temperature, in the operating room and intensive care ward."

[5] Administração Central do Sistema de Saúde, "Manual de Normas de Enfermagem, Procedimentos Técnicos”, ACSS, $2^{\circ}$ Edição, Lisboa 2011, http://www.acss.min-saude.pt/

\section{AUTHORS}

C. Rosa, A. Costa, E. Fernandes, F. Cardoso, P. S. Guimarães, J. Alves, I. Silva \& M. A. Marques are with Instituto Superior de Engenharia do Porto - School of Engineering, R. Dr. Antonio Bernardino de Almeida, 431, 4200-072 Porto (e-mail: \{1100860; 1090248; 1091422; 1080341; psg; jaa; ips; mmr\}@ isep.ipp.pt 